PRAMANA

- journal of

physics (c) Indian Academy of Sciences

pp. $1-11$

\title{
Quark-Gluon Plasma: Status of Heavy Ion Physics
}

\author{
R. V. Gavai \\ Department of Theoretical Physics, Tata Institute of Fundamental Research, Mumbai 400005.
}

\begin{abstract}
Lattice quantum chromodynamics (QCD), defined on a discrete space time lattice, leads to a spectacular non-perturbative prediction of a new state of matter, called quark-gluon plasma (QGP), at sufficiently high temperatures or equivalently large energy densities. The experimental programs of CERN, Geneva and BNL, New York of relativistic heavy ion collisions are expected to produce such energy densities, thereby providing us a chance to test the above prediction. After a brief introduction of the necessary theoretical concepts, I will present a critical review of the experimental results already obtained by the various experiments in order to examine whether QGP has already been observed by them.
\end{abstract}

\section{Introduction}

As is well known [1], the standard model of particle physics, $S U(3)_{c} \times S U(2)_{w} \times U(1)_{Y}$ broken spontaneously to $S U(3)_{c} \times U(1)_{e m}$, has been tested with great precision at LEP. All these tests rely heavily on the fact that the corresponding coupling is weak and hence the usual weak coupling perturbation theory can be employed in deriving the required theoretical predictions. Since the electromagnetic and weak couplings are indeed rather small in the currently accessible energy range, $\alpha_{e m} \simeq 7.3 \times 10^{-3}$ and $\alpha_{w} \simeq 3.4 \times 10^{-2}$, use of perturbation theory is not a serious limitation in the precision tests of the electroweak theory. However, the strong interaction coupling, $\alpha_{s}$, is (i) a strongly varying function of energy in the same range, (ii) about 0.11 at the highest energy at which it has been measured so far, and (iii) $\sim 1$ at a typical hadronic scale. Therefore, testing the strongly interacting sector of the standard model using only perturbation theory is a major shortcoming of the precision tests of the standard model.

Formulating quantum chromodynamics (QCD), which is an $S U(3)$ gauge theory of quarks and gluons, on a discrete (Euclidean) space-time lattice, as proposed by Wilson [2], and simulating it numerically as first shown by Creutz [3], one can obtain [4] several post-dictions of QCD in the non-perturbative domain of large $\alpha_{s}$. These include qualitative aspects, such as quark confinement and chiral symmetry breaking, and quantitative details such as hadron masses and their decay constants. While these agree with the known experimental results within the sizeable theoretical errors, it is fair to say that no serious experimental test of any non-perturbative prediction of QCD has so far been made. Relativistic heavy ion collisions offer a great window of opportunity to do so. Application of lattice techniques to finite temperature QCD has resulted in the prediction [5] of a new state of matter, called Quark-Gluon Plasma(QGP), at sufficiently high temperatures or energy densities. Chiral symmetry, broken spontaneously at zero temperature, seems to be restored in 
this new phase characterised by a much larger degrees of freedom characteristic of "free" quarks and gluons. Nevertheless, the phase appears to be inherently non-perturbative in the experimentally interesting range of $1 \leq T / T_{c} \leq 4-10$, where $T_{c} \sim 150 \mathrm{MeV}$ is the transition temperature at which the energy density varies most rapidly. The energy density, $\varepsilon$, in this range is $15-20 \%$ smaller [6] than the value of the corresponding ideal gas of quarks and gluons whereas a maximum of 3-5\% deviation is allowed for a weakly interactive perturbative QGP. While the precise values for $\varepsilon$, or $T_{c}$, as well as the nature of the phase transition (whether first order or second) depend on the number of light quark flavours, the quoted values above being for 2 flavours of mass about $15 \mathrm{MeV}$, many simulations with varying numbers of light flavours suggest that an energy density greater than $1 \mathrm{GeV} / \mathrm{fm}^{3}$ is needed to reach the QGP phase.

Collisions of heavy ions at very high energies can potentially produce regions with such large energy densities. Furthermore, since the transverse size of such regions is given by the diameter of the colliding nuclei, one can hope that these collisions will satisfy the necessary thermodynamical criteria of large volume $\left(L \sim 2 R_{A} \gg \Lambda_{Q C D}^{-1}\right)$ and many produced particles. A crucial unanswered question is whether thermal equilibrium will be reached in these collisions, and if yes, when it will be reached and how. Many different attempts have been made, and are being made, to address these issues. Here we will follow Bjorken's picture as it is most widely used in the field. Bjorken argued [7] that for sufficiently high energies, $\sqrt{s}>15 A \mathrm{GeV}$ where $\sqrt{s}$ is the total CMS energy of the two colliding nuclei of mass number $A$, the nuclei bore through each other and leave behind a baryonless blob of produced particles in the center (around $y_{c m}=\frac{1}{2} \ln \left[\left(E+P_{L}\right) /\left(E-P_{L}\right)\right] \sim 0$ ). After an equilibration time $\tau_{0}$, the energy density in the blob was estimated by Bjorken to be

$$
\varepsilon=\frac{1}{\mathcal{A} \tau_{0}} \cdot \frac{d E_{T}}{d y},
$$

where the effective area $\mathcal{A}=\pi R_{A}^{2}=3.94 A^{2 / 3} \mathrm{fm}^{2}$ and $d E_{T} / d y$ is the measured transverse energy per unit rapidity round $y_{c m} \approx 0.0$.

The Bjorken scenario for how the (thermally) equilibrated blob evolves is also the backbone of the analyses seeking to extract information from the data on whether QGP did form in the heavy ion collisions. According to this scenario, the hot blob cools by expanding and the matter in it goes through various stages such as QGP, mixed phase and a hadron gas, depending on the initial energy density reached and the equation of state. A further rapid expansion of the hadron gas leads to such large mean free paths for the hadrons that they essentially decouple from each other. If this freeze-out is sufficiently fast, the freestreaming hadrons, $\pi, k, \cdots$ etc. will, however, retain the memory of the thermal state from which they were born by having thermal momentum distributions. Thus the information from observables related to light hadrons can tell us about the temperature at this 'thermal freeze-out' and the velocity of expansion. To get a glimpse at still earlier times, one has to turn to 'harder' probes which typically involve a larger scale such as masses of heavy quarkonia, as we will see below.

The Bjorken scenario is for very high collision energies, when one expects to obtain a baryon-free region, and Eq. 1 is a valid description. The present collision energies may not be sufficient for it to hold, i.e., there may be a lot of baryons deposited in the central region of $y_{c m} \approx 0.0$. A reliable analogue of Eq. 1 is however not available in that case. Note that even the theoretical estimate from lattice QCD above was for a baryonless case. In addition to temperature, one can also imagine increasing the baryon density of the strongly 
interacting matter or equivalently increasing the baryonic chemical potential $\mu_{B}$ and obtain a baryon-rich plasma. In principle, one knows how to handle the case of a nonzero baryon density on the lattice but it has so far turned out to be difficult in practice. Usual lattice techniques fail for nonzero $\mu_{B}$ due to technical reasons [5] and attempts to overcome [4] these have not been successful either. No reliable lattice estimates are therefore available in that case. Using models based on underlying symmetries, it has been recently argued [8] that the $T-\mu_{B}$ phase diagram of QCD with realistic quark mass spectrum should have a critical point at a nonzero $\mu_{B}$. The analysis of heavy-ion data by Ref. [8] did not reveal any such critical point. By varying the energy of the colliding beam of heavy ions, one may hope to unearth such a critical point. While upcoming experimental runs at CERN at $\sqrt{s} \sim 9 \mathrm{~A} \mathrm{GeV}$ will look for such a critical point, the model considerations above are inadequate to provide reliable information on the energy density of the QGP phase for nonzero $\mu_{B}$. Thus a greater theoretical effort is required to firm up the QCD prediction for the energy density for nonzero $\mu_{B}$ and also to obtain the analogue of Eq. 1. Of course, one can in stead go for higher energies to test QCD, where one expects to obtain a baryon-free region, making both the lattice estimate and Eq. 1 more accurate descriptions.

\section{Results from CERN}

The experimental programs of high energy heavy ion collisions are being pursued actively at present in Brookhaven National Laboratory (BNL), New York and CERN, the European Laboratory for Particle Physics, Geneva. $A u$ - $A u$ collisions at $\sqrt{s}=4.7 A \mathrm{GeV} \simeq 0.92$ $\mathrm{TeV}$ have been studied at BNL while $\mathrm{Pb}-\mathrm{Pb}$ collisions at $\sqrt{s}=17.3 \mathrm{~A} \mathrm{GeV} \simeq 3.6 \mathrm{TeV}$ have been investigated at SPS, CERN using beams of gold ions at $2.1 \mathrm{TeV} / \mathrm{c}$ and lead ions at 32.9 TeV/c respectively. Earlier sulphur beam at $6.4 \mathrm{TeV} / \mathrm{c}$ was used on sulphur and uranium targets at SPS, CERN and those results form a benchmark over which several aspects of $\mathrm{Pb}-\mathrm{Pb}$ collisions have been compared. I will focus largely on the latter since they correspond to the highest $\sqrt{s}$ used so far. Due to space restrictions, I will also have to restrict myself to highlights and I have to refer the reader for more details to the proceedings of Quark Matter conferences [9].

\subsection{Initial Energy Density}

The NA49 experiment reported measurements on $d E_{T} / d \eta$ quite a while ago [10] and reported $d E_{T} / d y \simeq 405 \mathrm{GeV}$ for $P b-P b$. Using a canonical guess of $1 \mathrm{fm}$ for the formation time, one obtains from Eq. 1

$$
\varepsilon_{B j}^{P b-P b}(1 \mathrm{fm})=2.94 \pm 0.3 \mathrm{GeV} / \mathrm{fm}^{3},
$$

which is certainly above the characteristic QGP-phase values from lattice QCD mentioned in sec. 1. Since appreciable numbers of baryons at $y_{c m} \sim 0$ have been observed at SPS, it is doubtful that the current energies are high enough for creating a baryon-free region assumed for Eq. 1. Nevertheless, Eq. 1 has been frequently used to estimate the energy densities achieved up to now. One has to be cautious therefore and make sure that other independent estimates are also similar and they do appear to be so.

Pramana - J. Phys., 


\subsection{Hadron Yields}

Assuming that a thermal freeze-out is triggered by a rapid expansion, one expects the momentum spectra of various hadrons to reflect the freeze-out temperature, $T_{f o}$, which will be blue-shifted by the collective expansion. For small transverse momenta, $p_{T} \ll m$, we expect the inverse slope of transverse mass distribution, $d \sigma / d\left(m_{T}-m\right)$ with $m_{T}^{2}=p_{T}^{2}+m^{2}$, to be given by $T_{\text {slope }}=T_{f o}+\frac{1}{2} m\left\langle v_{T}\right\rangle^{2}$. Thus one expects, $T_{\text {slope }}$ to vary linearly with the mass of the observed particle, $m$. Fig. 1 displays the dependence of $T_{\text {slope }}$ on $m$ for various particles produced in the $P b-P b$ collisions in the different CERN experiments. A clear linear rise is evident for most of them except the heavier strange particles for which freezeout may be occurring somewhat earlier. The $T_{f o}$ can be obtained from the intercept in Fig. 1 , while the average collective velocity can be obtained from the slope.

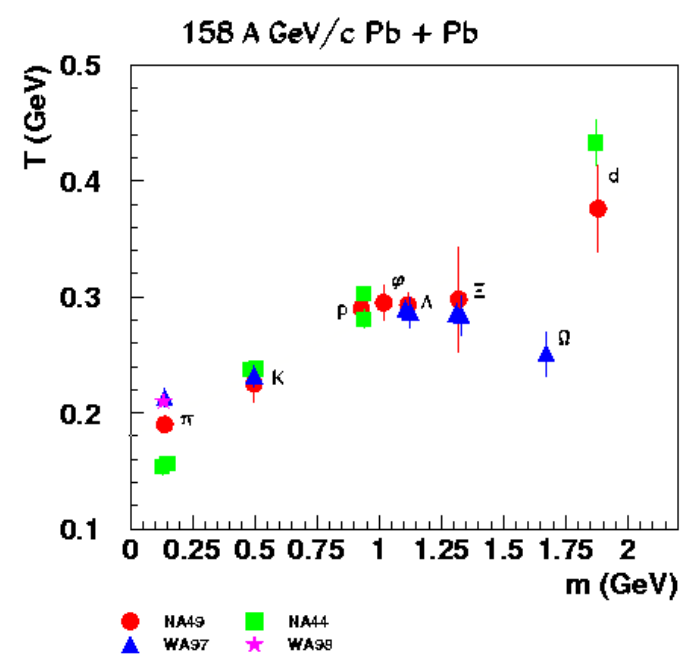

Figure 1. $T_{\text {slope }}$ for various particles as a function of mass.

Strangeness changing - chemical- reactions are typically slower than the elastic processes and hence are expected to freeze-out before the thermal freeze-out. The temperature and chemical potential at this freeze-out decides the particle yields of various types, provided these yields are measured for the full $4 \pi$-integrated region; otherwise the measurements will depend upon the details of the collective flow mentioned above. Furthermore, taking ratios of such yields, one can reduce the dependence on the collective dynamics even more. A simple thermal model of free particles at a temperature $T$, volume $V$ and chemical potential $\mu_{B}$ has been shown [11] to describe beautifully 22 ratios of particle yields which vary by three orders of magnitude, leading to $T_{f o}^{c h e m} \simeq 170 \mathrm{MeV}$ and $\mu_{B, f o}^{c h e m} \simeq 270 \mathrm{MeV}$. Fig. 2 displays the thermal and chemical freeze-out points for the SPS $P b-P b$ collisions along with those of other experiments. The figure is taken from Ref. [12] where the references for the data are also given. A comment about $\mu_{B, f o}^{\text {thermal }}$ may be in order, as we discussed above the corresponding $T_{f o}$ only. Since chemical equilibrium is lost earlier, it is strictly 


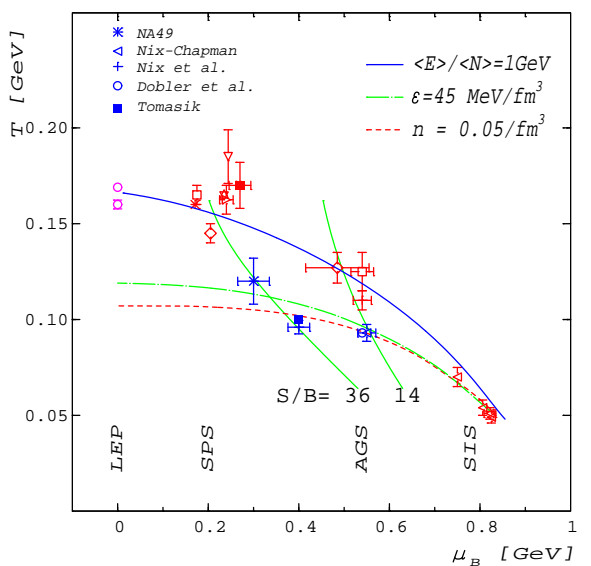

Figure 2. Chemical and thermal freeze-out points in the $\left(T, \mu_{B}\right)$ plane for various experiments. Taken from Ref. [12].

speaking not well defined. One simply adjusts $\mu_{B, f o}^{\text {thermal }}$ such that the particle ratios at $T_{f o}^{\text {therm }}$ agree with the observed values.

Since $T_{f o}^{c h e m}$ turns out to be very close to that expected for the quark-hadron transition from lattice QCD, it is plausible that the hadronic chemical equilibrium is a direct consequence of a pre-existing state of uncorrelated quarks and antiquarks and not due to hadronic rescatterings/reactions, since there is not much time for the latter. Hadron formation is then governed by the composition of the earlier state in a statistical manner and an expansion later does not change their yields. Needless to say though, the proximity of the two temperatures mentioned above is merely indicative. Indeed such temperatures and chemical potentials could still be reached via an expanding hadron gas as well. One then would expect though that the particle ratios will not reflect the underlying quark symmetries described next.

\subsection{Excess Strangeness}

Since the early days of heavy ion collisions, when sulphur beams at $6.4 \mathrm{TeV}$ were bombarded on sulphur targets, a global enhancement of strangeness in these collisions has been observed relative to $e^{+} e^{-}$or $p p$ collisions. Defining [13] a parameter $\lambda_{s}$ to count the strangeness, $\lambda_{s}=2\langle s+\bar{s}\rangle /(\langle u+\bar{u}\rangle+\langle d+\bar{d}\rangle)_{\text {produced }}$, it was found that $\lambda_{s}^{A A} \simeq 2 \lambda_{s}^{P P}$. For both $S(6.4 \mathrm{TeV})+A g$ and $P b(33 \mathrm{TeV})+P b$, a similar factor of 2 enhancement was observed. This global enhancement together with the picture of statistical hadron formation discussed above suggests an interesting pattern for specific particles.

Fig. 3 shows the results from WA97 for specific enhancements of $\Omega, \bar{\Xi}, \bar{\Lambda}$ etc. The 


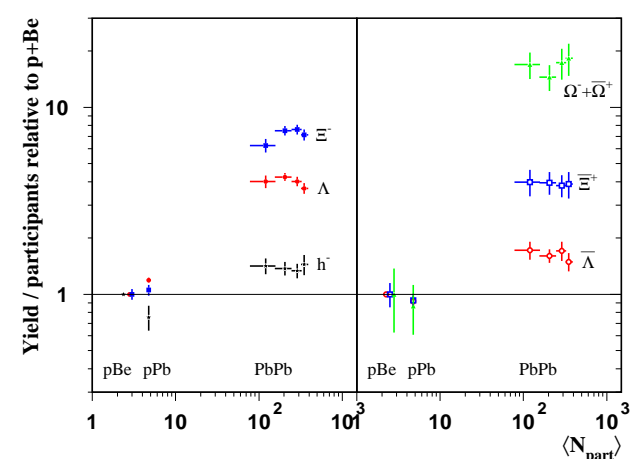

Figure 3. Specific strangeness enhancement in $\mathrm{Pb}-\mathrm{Pb}$ collisions seen by the WA97 collaboration [14] as a function of number of participants in the collisions.

yield for these particles per participant nucleon for $P b-P b$ relative to that in $p+B e$ is displayed as a function of average number of participants. The number of participants is an indicator of how central (or violent) the collision is; maximally central events are expected to have largest energy density deposited. One sees the yields to be almost independent of the centrality reached experimentally so far. Assuming now that the global strangeness enhancement by a factor of two indicates the relative probability of finding a strange quark in $P b-P b$ vs $p-B e$ to be twice, due perhaps to a formation of a QGP-state in the latter which evaporates just before the observed chemical freeze-out, one expects a factor of two enhancement for every extra strange quark (or antiquark) in a hadron, explaining the pattern in Fig. 3. Note that the masses of these particles increase as more strange quarks are added, $m_{\Omega}>m_{\Xi}>m_{\Lambda}$. Their production in a purely hadron rescattering/reactions scenario, therefore, will be subject to increasingly higher thresholds, resulting in an opposite pattern to that observed. Thus, the pattern in Fig. 3 of specific enhancements of strangeness clearly points to quark degrees of freedom. It will be interesting to see whether this enhancement sets in smoothly or abruptly as the number of participants vary.

\subsection{Excess Low Mass Dileptons}

An interesting anomaly, observed first in $S(6.4 \mathrm{TeV})+A u$ collisions by NA45/CERES [15] and confirmed for $P b(33 \mathrm{TeV})+A u$ collisions [16], is an enhancement of $M_{e^{+} e^{-}}$spectrum in the region $250 \mathrm{MeV}<M_{e^{+} e^{-}}<700 \mathrm{MeV}$. The data for $p(450 \mathrm{GeV})+A u$ collisions in the entire range $0 \leq M_{e^{+}} e^{-}<1500 \mathrm{MeV}$ can be explained by including the contributions 
from all known hadron decays of $\pi^{0}, \eta, \omega, \rho, \phi, \eta^{\prime}$ etc. within the acceptance of the detector. A similar exercise for $S+A u$ or $P b+A u$ reveals an enhancement in the low mass region mentioned above, with the enhancement factor being $2.6 \pm 0.5$ (stat) \pm 0.6 (syst). Various theoretical explanations have been offered [17] to explain it. For us, it is interesting to note that thermal emission from an expanding fireball with parameters similar to those in sec. 2.2. can account for the excess, thus supporting to the picture discussed there.

\section{$2.5 \mathrm{~J} / \psi$ Suppression}

As remarked in the introduction above, one needs to employ 'harder' probes to explore the physics of the fireball at earlier times when QGP may have existed. Production of $J / \psi$ is one such hard probe. Since it is a tightly bound meson of charm and anticharm quarks, Matsui and Satz [18] argued that color Debye screening of these heavy quarks will prevent formation of $J / \psi$, if QGP is formed in the heavy ion collisions. Due to a finite size and lifetime of the fireball, the observable effect is expected to be a suppression in the production of $J / \psi$. The NA38 and NA50 collaborations [19] measured $J / \psi$ cross sections for a variety of collisions, starting from $p+d$ to $P b+P b$ using the same muon spectrometer in the same kinematic domain $\left(0 \leq y_{\mu^{+} \mu^{-}}^{c m} \leq 1\right.$ and $\left.\left|\cos \Theta_{c s}\right| \leq 0.5\right)$. While the systematic errors are thus minimised, the lighter beams were necessarily of high energies; $\sqrt{S_{N N}}$ thus varies from $17 \mathrm{GeV}$ to $30 \mathrm{GeV}$. From the observed dimuon spectrum, $d N / d M_{\mu^{+} \mu^{-}}$, obtained after subtracting the background due to like-sign dimuons, the $J / \psi$ cross section was obtained by a fit of the observed spectrum for $M_{\mu^{+} \mu^{-}}>2.9 \mathrm{GeV}$ with 5 parameters: the number of events of Drell-Yan continuum, $J / \psi$, and $\psi^{\prime}$ and the $J / \psi$ mass and width.

Comparing $\sigma_{o b s}^{D Y}$ with $\sigma_{L O, t h}^{D Y}$, where isospin corrections were taken into account, the $K$ factor was found to be universal in $p p, p A$ and $A B$ collisions: $\sigma_{A \cdot B}^{D Y} \propto A \cdot B$ for all of them, where $A$ and $B$ are the mass numbers of the projectile and target respectively. Normalizing $B_{\mu^{+} \mu^{-}} \sigma_{A B}^{J / \psi}$ by dividing by $A \cdot B$ therefore, where $B_{\mu^{+} \mu^{-}}$is the branching fraction of $J / \psi$ in to $\mu^{+} \mu^{-}$, one could expect QGP formation to be signalled by a drop at some value of $A \cdot B$. Fig. 4 shows the NA38 and NA50 results where one notices a gradual fall in with $A \cdot B$ for all values. Note that some measurements have been re-scaled so that all are for the same energy in this figure. The decreasing cross section for all values of $A \cdot B$, including small ones, is an indication of the presence of yet another mechanism for $J / \psi$-suppression in these collisions. Thus any suppression due to QGP will have to be over and above this 'normal suppression'.

Production of heavy quarkonia is an old and mature area of perturbative QCD. In particular, hadroproduction of $J / \psi$ has been explained both in the colour evaporation model [20] and the colour octet model [21] at $\sqrt{s}$ comparable to those in Fig. 4. So it is a natural question to ask whether the decrease in Fig. 4 can be explained using pQCD. Unfortunately, sufficient information on the nuclear structure functions, especially the gluonic ones, is not available at present; assuming them to be independent of mass number $A$ or $B$ is perhaps incorrect in view of the famous EMC-effect. Using the existing models of the EMC-effect, on the other hand, one finds hardly any decrease in the cross section in Fig. 4. It is even

likely that this lack of decrease of $B_{\mu^{+} \mu^{-}} \sigma_{A B}^{J / \psi} / A B$ with $A B$ is a generic feature independent of the models of the EMC effect. This is due to the fact that the dominant contribution to the cross section in Fig. 4 comes from the so-called antishadowing region in $x$ which is 


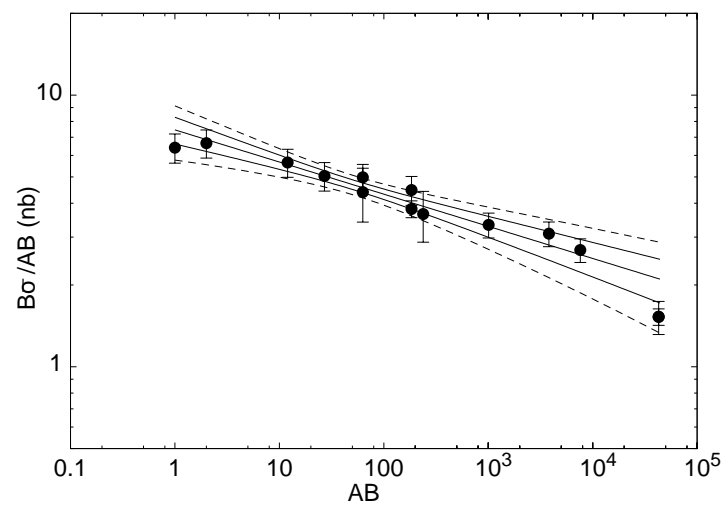

Figure 4. $J / \psi$ cross section times its branching fraction in to dimuons as a function of product of the mass numbers of target and projectile, AB. The data are from Ref. [19] and the $1 \sigma$ (full lines) and $2 \sigma$ (dashed lines) curves are from Ref. [23].

hard to avoid for even the gluons due to the momentum sum rule. In view of the continuous decrease in Fig. 4, i.e. even for $p+$ light- $A$, where the radius of the target is only 2-4 times larger than that for the hadroproduction [20,21], one has to ask whether a pQCD description of total cross sections for $J / \psi$ is at all possible. It would be interesting and desirable to thrash out this question by extensive investigation of the nuclear glue and its impact on the $J / \psi$ cross section.

The normal suppression in Fig. 4 has been explained [22] as a final state interaction. The produced $J / \psi$-state or its precursor can get absorbed in the nuclear matter (of the target and beam). Treating $\sigma_{a b s}^{\psi N}$ as a free parameter and using the known nuclear profiles, one finds that a $\sigma_{a b s} \sim 6.4 \mathrm{mb}$ can explain the linear fall in Fig. 4 quantitatively in Glauber type models. However, the $P b-P b$ data point seems to be off this linear fall, and exhibits thus an 'anomalous suppression'. One can alternatively use an empirical $(A B)^{\alpha}$ fit to all points except the $P b-P b$, which too will be linear on the scales of Fig. 4 and the $P b-P b$ data point stands out again.

Unfortunately, the issue of how statistically significant this anomalous suppression is gets affected by the crudeness of the theory described above as well as by the assumptions needed to rescale some of the data points. Ignoring these systematical theoretical errors, one finds the anomalous suppression to be a $5 \sigma$ effect [19], while including them leads [23] to a conclusion that no anomalous suppression exist at a $2 \sigma$ or $95 \%$ confidence level, as shown by the $2 \sigma$-band (enclosed by dashed lines) in Fig. 4 .

The NA50 collaboration also measures $J / \psi$-suppression as a function of the total produced transverse energy $E_{T}$. Using the same procedure as outlined above, the number of $J / \psi$ events and the Drell-Yan events ${ }^{1}$ in each $E_{T}$-bin are determined. By taking their ra-

\footnotetext{
${ }^{1}$ Note that the usual Drell-Yan cross section in pQCD is defined only by integrating all $E_{T}$.
} 
tio, one obtains a less systematic error prone $R_{\text {expt }}=B_{\mu^{+} \mu^{-}} \sigma^{J / \psi} / \sigma_{M_{1}-M_{2}}^{D Y}$ as a function of $E_{T}$, where $M_{1}-M_{2}$ is the range of dimuon mass over which the Drell-Yan cross section is integrated. Using simple geometrical models, $E_{T}$ can be related to the impact parameter $b$ at which the two nuclei collide. Furthermore, any given $b\left(E_{T}\right)$ can be related to an average nuclear path length $L$ which the produced $J / \psi$ (or its precursor) has to traverse and which will determine the probability of its absorption in nuclear matter.

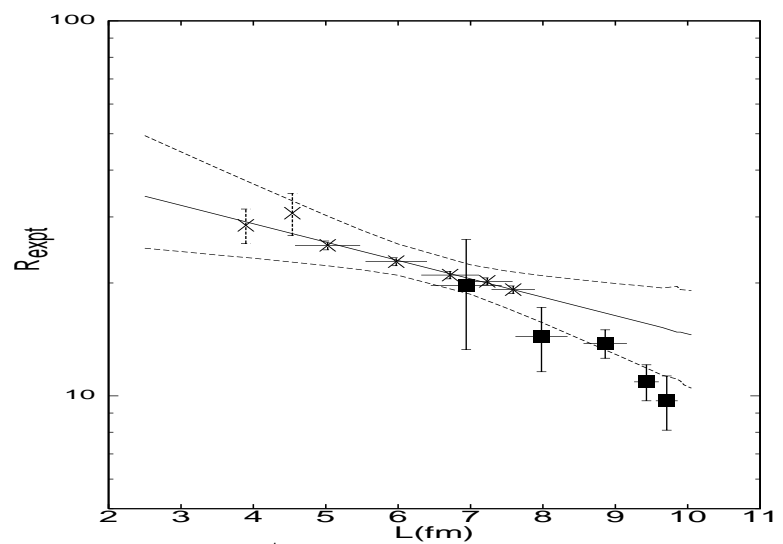

Figure 5. The ratio of $J / \psi$ cross section and the Drell-Yan cross section vs. $L$ in $\mathrm{fm}$. The crosses are NA38 data, shown along with the straight line fit, a $4 \sigma$ band [24] around it, and the NA50 data (squares) with $4 \sigma$ errors on them. The data are from Ref. [19].

Fig. 5 shows $R_{\text {expt }}$ as a function of $L$, as determined by the NA50 collaboration, using $M_{1}$ $=2.9$ and $M_{2}=4.5$. The normal nuclear suppression can be well approximated by $R_{\text {expt }}=$ $A \cdot \exp \left(-\rho_{\text {nucl }} \cdot \sigma_{a b s} \cdot L\right)$ or can be calculated more exactly in a Glauber model. The straight line in Fig. 5 displays the fit for the light nuclei for $\rho_{\text {nucl }}=0.17 / \mathrm{fm}^{3}$ and $\sigma_{a b s} \simeq 6.6 \mathrm{mb}$. The low $L$ point for $P b-P b$ collisions, corresponding to peripheral collisions, falls on the fitted line while all the large $L$ points fall below it. Again, one can ask for the statistical significance of this anomalous behaviour. Since the fit above uses data from $E_{T}$-bins, or equivalently $L$-bins, for lighter nuclei, there are again sizeable errors on the theoretical prediction. For the 1995 data, which seem broadly in agreement with the 1996 data and the 1998 data, it has been estimated [24] that all the $\mathrm{Pb}-\mathrm{Pb}$ data points fall in a $4 \sigma$-band although they are all systematically below the theoretical prediction, as shown in Fig. 5.

It seems thus likely that an additional mechanism to suppress $J / \psi$ production in $\mathrm{Pb}-\mathrm{Pb}$ collisions, especially at large values of transverse energy $E_{T}$, or large $L$, is needed over and above the normal suppression caused by absorption in the surrounding nuclear matter. There have been several theoretical attempts to provide such a mechanism including, of course, invoking a possible a quark-hadron transition. A key non-QGP scenario invokes the possibility of destruction of the $J / \psi$ by the so-called co-mover debris of the collisions. In a recent ${ }^{2}$ such work, it has been claimed [25] that the entire NA50 $E_{T}$-spectrum of

\footnotetext{
${ }^{2}$ Although this came after my talk, I include it here for completeness.
} 
the $J / \psi$ cross section ratio $R$ can be explained using the co-mover picture. In fact, the second shoulder in the $E_{T}$-behaviour observed [26] in the 1998 data and which could be due to QGP [27], has been explained in Ref. [25] as due to fluctuations at the tail of the $E_{T}$-spectrum. The difference between this mechanism and a QGP model will, therefore, most likely show up at the upcoming RHIC collider in BNL where $A u(19.7 \mathrm{TeV})+A u$ $(19.7 \mathrm{TeV})$ collisions will be studied this year and the $E_{T}$ tail will extend much farther.

\section{Conclusions and Outlook}

An important non-perturbative prediction of (lattice) QCD is the existence of a new phase of matter, Quark-Gluon Plasma, at sufficiently high temperatures. Since the Standard Model has so far been tested experimentally only in the weak coupling regime, it seems desirable to confront this prediction with experiments. Collisions of heavy ions at very high energy may be able to deposit the required high energy density over a reasonable volume. The experimental programs at BNL, New York and CERN, Geneva have by now provided results for $A u$ on $A u$ and $P b$ on $P b$ at $\sqrt{s} \simeq 0.9 \mathrm{TeV}$ and $3.6 \mathrm{TeV}$ (or $\sqrt{s}_{N N} \simeq 5 \mathrm{GeV}$ and $17 \mathrm{GeV}$ ) respectively. The year 2000 should witness a factor of about 39 increase in the colliding CMS energy at BNL while LHC at CERN should achieve a $\sqrt{s}=1150 \mathrm{TeV}$. The experiments so far have provided tantalizing hints of the new phase and therefore of the exciting physics in the years ahead.

A fireball of QGP produced in these collisions cools by expanding and converts into ordinary hadrons and leptons fairly quickly. Since this makes a distinction of events with QGP formation from those without it a very tough task, it seems prudent to look for a congruence of various signatures in as many different ways of detecting QGP as possible. Interestingly, the current results do indicate such a trend of congruence from apparently unrelated measurements.

Soft hadron production data, such as $p_{T}$-distributions, two particle (HBT) correlations and ratios of particle yields, can be interpreted in terms of a chemical freeze-out, followed by a thermal freeze-out. The freeze-out temperature for the former for the CERN SPS data turns out to be $\sim 170 \mathrm{MeV} \simeq T_{c}$ (quark-hadron transition), suggesting that the hadrons were formed from an uncorrelated QGP-like state. The global excess of strangeness by a factor of two and the specific enhancement pattern of $\Omega, \bar{\Xi}$ and $\bar{\Lambda}$, seen by the WA97 experiment, showing larger enhancement for the heavier particles with more strange quarks is consistent with this picture. In fact, the increasing thresholds make it very difficult to explain this pattern in any conventional hadronic picture. Thermal effects arising from $T \sim$ $120 \mathrm{MeV}$, the thermal freeze-out temperature, can account for the observed excess of low mass dielectron events of the NA45 experiment.

Finally, anomalous $J / \psi$ suppression seen by the NA50 experiment for $P b-P b$ collisions can be understood as arising out of a deconfined quark-gluon plasma. Nevertheless, much more theoretical and experimental work will be needed to make a convincing case of quarkgluon plasma formation in the heavy ion experiments since the signals are still not spectacular in their statistical significance and credible alternative explanations exist in many

cases for the observed results . Clearly, the commissioning of RHIC will be a big boost and will hopefully result in making a definitive case for quark-gluon plasma. 


\section{References}

[1] See, e.g., Review of Particle Physics, Eur. Phy. J., C3, 81 (1998).

[2] K. G. Wilson, Phys. Rev. D10, 2445 (1974).

[3] M. Creutz, Quarks, gluons and lattices, Cambridge Mongraphs (1983).

[4] Proceedings of a) Lattice 98, Nucl. Phys. B(PS)73, 1999, b) Lattice 97, Nucl. Phys. B(PS)63, 1998 etc.

[5] For a review, see, R. V. Gavai in "Quantum fields on the computer", Ed. M. Cruetz, World Scientific 1992, p. 51.

[6] C. Bernard et al., Phys. Rev. D55, 6861 (1997).

[7] J. D. Bjorken, Phys. Rev. D27, 140 (1983).

[8] M. Stephanov, K. Rajagopal, and E. Shuryak, Phys. Rev. D60, 114028 (1999).

[9] Proceedings of a) Quark Matter 99, Nucl. Phys. A661, 1999, b) Quark Matter 97, Nucl. Phys. A638, 1998 etc.

[10] NA49 Collaboration, T. Alber et al., Phys. Rev. Lett. 75, 3814 (1995).

[11] P. Braun-Munziger, I. Heppe and J. Stachel, Phys. Lett. B465, 15 (1999).

[12] J. Cleymans and K. Redlich, Phys. Rev. Lett. 81, 5284 (1998).

[13] F. Becattini, M. Gaździcki and J. Sollfrank, Eur. Phys. J. C5, 143 (1998).

[14] WA97 Collaboration, E. Andersen et al, Phys. Lett. B449, 401 (1999).

[15] CERES Collaboration, G. Agakichiev et al, Phys. Rev. Lett. 75, 1272 (1995).

[16] CERES Collaboration, G. Agakichiev et al, Phys. . Lett. B422, 405 (1998); B. Lenkeit in a) of Ref. [9].

[17] R. Rapp in a) of Ref. [9].

[18] T. Matsui and H. Satz, Phys. Lett. B178, 416 (1986).

[19] NA50 Collaboration, M. C. Abreu et al, Phys. Lett. B450, 456 (1999); ibid, B410, 337 (1997).

[20] R. V. Gavai, D. Kharzeev, H. Satz, G. A. Schuler, K. Sridhar and R. Vogt, Int. J. Mod. Phys. A10, 3043 (1995).

[21] S. Gupta and K. Sridhar, Phys. Rev. D54, 5545 (1996).

[22] A. Capella, J. A. Casado, C. Pajares, A. V. Ramallo and J. Tran Thanh Van, Phys. Lett. B206, 354 (1988); C. Gerschel and J. Hüfner, Phys. Lett. B207, 253 (1988); Z. Phys. C56, 171 (1992).

[23] R. V. Gavai and S. Gupta, Phys. Lett. B408, 397 (1997).

[24] R. V. Gavai, Mod. Phys. Lett. A14, 821 (1999).

[25] A. Capella, E. G. Ferreiro and A. B. Kaidalov, hep-ph/0002300.

[26] NA50 Collaboration, M. C. Abreu et al, CERN-EP-2000-013, Phys. Lett. B, in press.

[27] S. Gupta and H. Satz, Phys. Lett. B283, 439 (1992); H. Satz, Nucl. Phys. A661, 104c (1999). 